\title{
Urgences
}

\section{Signé Laforgue : un Hamlet sur mesure}

\section{Sophie Lemieux}

Numéro 25, octobre 1989

Multiples de Hamlet

URI : https://id.erudit.org/iderudit/025541ar

DOI : https://doi.org/10.7202/025541ar

Aller au sommaire du numéro

Éditeur(s)

Urgences

ISSN

0226-9554 (imprimé)

1927-3924 (numérique)

Découvrir la revue

Citer cet article

Lemieux, S. (1989). Signé Laforgue : un Hamlet sur mesure. Urgences, (25),

22-29. https://doi.org/10.7202/025541ar d'utilisation que vous pouvez consulter en ligne.

https://apropos.erudit.org/fr/usagers/politique-dutilisation/ 


\section{Signé Laforgue: un Hamlet sur mesure Sophie Lemieux}

Soit Hamlet comme une substance à laquelle William Shakespeare aurait donné la forme d'un cube Rubik, caméléon de surcroît.

Soient plusieurs manipulateurs qui, depuis, s'évertuent à déplacer ou replacer les rouages du cube Hamlet selon...

Parmi ceux-là, un certain Jules Laforgue, tout de noir vêtu pour l'occasion et marchant d'une allure correcte et traînarde, traînarde et correcte.

Or, le jeu du manipulateur Laforgue dédit son allure, tout comme dans la souricière de son Hamlet «le chant de triomphe de l'usurpateur Claudius se chante sur l'air des "pressentiments trompeurs" $" 1$.

- À Paris, Altesse, vous le savez, il y a pour votre légende spéciale, Paul Bourget qui la cultive et l'aggrave [...]; il y eut Arthur Rimbaud qui en est mort, après [...]; il y a moi qui vous prend en gaîté, Altesse, à la Yorrick [...], qui vous prend en gaîté parce que c'est plus fort que moi. 2

écrit Laforgue, dans "À propos de Hamlet».

En toute connaissance de cause, il choisit donc la gaîté comme règle du jeu. Digne d'un fou du roi et d'une shakespearienne mémoire, c'est une gaîté troublante, qui suppose une conscience de soi, mais qui mise sur l'ambiguïté et le paradoxe. Mais jugez plutôt.

On dit qu'un des premiers mouvements du bouffon Laforgue fut de se créer un Hamlet sur mesure: les mesures en question étaient évidemment les siennes, traits pour traits ${ }^{3}$.

1 Jules Laforgue, « Hamlet ou les suites de la piété filiale ", Moralités légendaires, Paris, Gallimard, coll. «Folio», 1977, p. 34 . A moins d'avis contraire, les références à cette nouvelle (et à cette édition) seront immédiatement suivies, entre parenthèses, du titre abrégé (HSPF) et du numéro de la (des) page(s).

2 Jules Laforgue, "À propos de Hamlet ", ibid., p. 208.

3 Sur la ressemblance physique entre Hamlet et Laforgue, voir Pascal Pia, “ Notes (Hamlet ou les suites de la piété filiale) ", ibid., p. 235-236. 
Façon de s'approprier le personnage ou inversement de s'identifier à lui 4; façon aussi de s'auto-ironiser. Peut-on, après tout, pratiquer l'hypertextualité ${ }^{5}$ sans être conscient de sa propre relativité, de sa propre fragilité?

Un Hamlet de moins; la race n'en est pas perdue, qu'on se le dise. (HSPF, p. 64)

conclut Laforgue. Au cimetière, Yorrick fait mine de lever son chapeau; sur scène, Laforgue reprend son jeu.

C'est un jeu toujours double, mettant en relief la relation hypertextuelle elle-même, tout en travaillant et transformant directement l'hypotexte.

Ainsi, dès le titre, "Hamlet ou les suites de la piété filiale », la nouvelle de Laforgue fait ouvertement référence à son hypotexte shakespearien. Le pacte hypertextuel est signalé, signé même.

Aussitôt, toutefois, il propose une interprétation: le "ou " qui unit «Hamlet » et « les suites de la piété filiale» suggérant moins un choix qu'une définition laforguienne du premier terme.

Définition décidément ambiguë, étant donné le mot "suites " qui, de «conséquences " à "prolongations ", peut aussi signifier «héritage" ou "hérédité " puisqu'il s'agit de piété filiale.

Et pour entretenir l'équivoque, Laforgue reprend en épigraphe le "C'est plus fort que moi". Aveu d'un besoin, d'un désir incontournables, en même temps que soumission à l'autorité. Mais quelle autorité? Celle du spectre paternel si le «moi» est hamlétien? du spectre hamlétien, si le «moi» est celui de Laforgue? le "ça» de la psychanalyse avant l'heure?

Rire de Laforgue, que l'Impératif Catégorique n'arrivera pas à saisir. Rire, parce que c'est peut-être la seule façon d'entrer en s'échappant. Rire, dire (comme dormir, mourir), sans ( $\left(s^{\prime}\right)$ être trop pris au sérieux.

4 Voir à ce sujet l'article de Jean-Luc Steinmetz, «Portrait d'Hamlet en Laforgue adulte ou l'art, le rat et le raté ", Silex, n 3 , Grenoble, 1977, pages 89 et 90 surtout.

5 Si le terme n'existait pas encore, la pratique, en revanche, existait déjà. J'utilise la terminologie et les concepts de Gerard Genette, Palimpsestes. La littérature au second degré, Paris, Seuil, coll. « Poétique ", 1982, 467 p. 
24

La temporalité et les personnages suggèrent aussi ce rapport ambigu à l'hypotexte.

Clin d'œil. Le temps de l'histoire, dans la nouvelle de Laforgue, est celui d'une mise en scène: 1601 , date probable de la première représentation du Hamlet de Shakespeare.

Temporalité ou convention pour rire, puisque perturbée par de nombreux anachronismes proximisants ${ }^{6}$ et même par un curieux phénomène inverse: la comédienne Kate songe à aller soigner les blessés de la guerre de Cent Ans ( $H S P F$, p. 58)!

Pied-de-nez, également. Alors que le temps de l'histoire coïncide, à peu de choses près, avec celui de l'écriture de l'hypotexte, les personnages, eux, retrouvent leurs noms préshakespeariens: Horwendill (Hamlet père), Gerutha (la reine), Fengo (le roi usurpateur) ${ }^{7}$.

En fait, ces personnages sont très peu présents dans la nouvelle (sauf dans les pensées de Hamlet, bien sûr). La reine et le roi n'apparaissent réellement que lors de la représentation de la souricière; le spectre majestueux et damné de l'hypotexte devient peinture en pied qui assène à son fils des clins d'œil royalement «coquins et faunesques" ( $H S P F$, p. 25).

Éliminés, les Rosencrantz, Guildenstern et les officiers de garde. Éliminé, ô ironie, l'intègre Horatio, porteur de confidences et de vérité. Disparus, d'entrée de jeu, Polonius et Ophélie... Et Laërtes, quant à lui, arrive de justesse mais néanmoins à point pour tuer Hamlet, à la fin.

Aux côtés de Hamlet, ne restent donc que les comédiens, auxquels Laforgue donne des noms (William [!], pour l'un et Kate, alias Ophélia [!], pour celle qui interprétera Batista dans la souricière)... et les fossoyeurs. Interlocuteurs de choix pour celui qui trouve "L'Art [...] si long et la vie si courte!» ( $H S P F$, p. 49)

Dans un décor qui prend au pied de la lettre le «il y a quelque chose de pourri dans le royaume" (à preuve, toutes les allusions à la putréfaction dans les trois premières pages

6 Anachronismes de fait: « douches " (HSPF, p. 25), “ cigarettes" (ibid., p. 30); de culture ou de pensee: mention de Hobbes (ibid., p. 27), de Paul Delaroche (ibid., p. 59), des « prolétaires" (ibid., p. 41); de langue: le cliché « public houleux $\leadsto$ (ibid., p. 58), etc.

7 Bien que Laforgue emprunte le “Claudius" de Shakespeare pour jouer dans la souricière. À ce sujet, voir Jean-Luc Steinmetz, op. cit., p. 90. 
de la nouvelle seulement), la vedette appartient au prince Hamlet.

Un Hamlet démystifié ou "aggravé" (dirait Genette) par Laforgue, toujours héros tourmenté, mais désormais si familier et si "petite crapule". Son hésitation est caricaturée: «Hamlet, homme d'action, perd cinq minutes à rêver» ( $H S P F$, p. 35). Ses gestes sont «démotivés» (Genette encore); il a tué Polonius pour se faire la main (HSPF, p. 35), il saccage la nature sans raison ( $H S P F$, pages 38-39), etc.

C'est un Hamlet conscient d'être une personnalité légendaire, qui s'étend avec un brin de complaisance sur sa future célébrité ( $H S P F$, p. 35-36) ou qui conclut, après avoir rêvé d'anonymat: "Ç'aura été, de toutes mes idées, la plus hamlétique " (je souligne, $H S P F$, p. 36 ).

Enfin, c'est un Hamlet dont le discours, par moments, parodie l'hypotexte ${ }^{8}$. Malgré les changements de noms, les personnages, et surtout Hamlet, sont donc bien ceux de Shakespeare.

À coup d'intrusions, le narrateur s'impose dans le récit, donnant ici des indications scéniques, commentant là les gestes et intentions de ses personnages ( $H S P F$, pages 45 et 46 , par exemple).

Ainsi, même s'il s'agit d'une narrativisation avec vocalisation de l'hypotexte (passage d'un texte dramatique à un texte narré au "je»), la nouvelle mime, dans son fonctionnement, le texte théâtral.

Jouant au régisseur ${ }^{9}$, le narrateur de Laforgue dissipe l'illusion narrative et montre encore une fois qu'il reprend et dirige, à sa manière, un texte et des personnages préexistants.

Mais son rôle n'est pas clair.

S'il dirige ses personnages, sa présence, à certains moments, est si envahissante qu'il devient difficile de distinguer son discours de celui de Hamlet:

8 Par exemple: “Stabilité ! Stabilité ! Ton nom est Femme... » (HSPF, p. 26); "Mais ne plus étre, ne plus y être, ne plus en etre !" (HSPF, p. 47), etc.

9 À ce sujet, voir l'analyse de Danielle Bajomée, "Hamlet, une moralité oubliée de Jules Laforgue ", Revue des langues vivantes, $n^{\circ} 4$, Bruxelles, 1967, p. 402-405 surtout. 
La nuit tombe! Ah! II faut agir! Hamlet reprend le chemin du château sans trop se laisser envahir [...]. (HSPF, p. 51)

Si l'histoire est connue, cela n'empêche pas le narrateur de s'étonner soudain du cours des événements de son propre récit: «(Non, non! Ce n'est pas possible! Cela s'est fait trop vite!) (HSPF, p. 60)

Si ce narrateur se nomme à la fin (HSPF, p. 62), peut-être aussi s'est-il mis en scène en la personne de l'Ange Gardien de Hamlet, nommé deux fois dans la nouvelle (HSPF, pages 28 et 39: "C'est fort commode: et si Hamlet n'en est pas encore à songer qu'il n'a guère autrement apprécié la triste Ophélie [...] son Ange Gardien n'en pense pas moins.")

Par ces astuces de vieux conteur, le narrateur parvient à s'insérer dans l'histoire comme personnage et témoin: c'est aussi SON histoire qu'il raconte.

De diverses façons, la narration, les personnages, la temporalité, l'épigraphe et le titre signifient donc au lecteur le "second degré" de la nouvelle. Multiples effets de distanciation qui autorisent la transformation de l'hypotexte. «N'importe qui peut pêcher avec le ver qui a mangé un roi / et manger le poisson qui a mangé le ver", affirme le Hamlet de Shakespeare ${ }^{10}$. Réponse goguenarde de Laforgue: «J'ai de l'infini sur les planches " (HSPF, p. 51).

L'histoire demeure toujours celle de Hamlet, prince du Danemark, telle qu'on la connait ou telle qu'on devrait la connaître ${ }^{11}$, mais elle est modifiée.

Une des accentuations percutantes de Laforgue consiste à faire de son prince un dramaturge en herbe, ce qu'il est déjà, mais à un degré moindre, chez Shakespeare. Étant d'abord et avant tout l'auteur de la souricière, Hamlet est maintenant bien plus préoccupé par des questions de réception, de création et d'art que par la vengeance de son père.

En outre, et c'est un autre mouvement percutant, Hamlet apprend, lors d'une sortie au cimetière, qu'il n'est par le fils de la reine Gerutha, mais bien le frère de Yorrick par sa

10 William Shakespeare, La tragédie d'Hamlet, prince de Danemark, Paris, Gallimard, coll. «Folio ", 1978, p. 152 (acte IV, scene 3).

11 Voir les commentaires de Gérard Genette, op. cit., p. 330. 
mère, une gypsie qui mourut à la naissance de Hamlet (de la césarienne qu'on dut alors pratiquer, $H S P F$, p. 44).

Laforgue pratique ainsi une démotivation majeure du personnage de Hamlet, qui perd les raisons profondes (ou psychologiques) justifiant son désir de punir la reine et le roi. Démotivation qui se change rapidement en motivation, puisque Hamlet se trouve un nouvel héritage: «Hamlet frère d'un fou de cour; il n'est donc pas si "fils de ses œuvres" qu'il le croyait !...» (HSPF, p. 44); et aussi : «Et par ma mère et par mon frère, et tout, j'étais damné d'avance» (HSPF, p. 49). Fou de cour, clown, jeu: chez Shakespeare, Hamlet saluait l'arrivée des comédiens en les appelant " mes maitres " (acte II, scène 2).

Si l'approbation des comédiens confirme à Hamlet son talent ${ }^{12}$, l'interprétation de Kate dans le rôle de Baptista lui ouvre des horizons nouveaux ( $H S P F$, p. 58).

La représentation de la souricière, chez Laforgue, est bel et bien un "piège-à-roi-fratricide"; mais surtout, elle met au monde un auteur: «Parbleu! Je n'étais qu'un écolier! Voilà ce qui me manquait, l'épreuve de la rampe" (HSPF, p. 58). Enfin, nouvelles données en main, Hamlet choisit (quel luxe) de s'enfuir avec Kate; au diable son trône! Prétextant un arrêt sur la tombe de son père, il va droit à celle d'Ophélie, où il est tué, comme il se doit, par Laërtes.

Finale surprenante, mais qui rétablit l'hypotexte. Plus d'Horatio, toutefois, pour tout raconter, mais une comédienne, Kate, qui interprétera cette finale, après avoir interprété l'œuvre de Hamlet.

Laforgue réduit donc l'hypotexte, dont il ne conserve que trois scènes (l'arrivée des comédiens, la représentation de la souricière, la scène du cimetière); il condense, fait des substitutions et des amplifications qui sont des interprétations.

L'obsession du narrateur à nommer «l'irrégulier décès" du père (quatre allusions dans les trois premières pages, déjà) traduit bien la hantise causée par l'apparition et les révélations du spectre, dans l'hypotexte.

De même, les dévotions (si l'on peut dire) de Hamlet aux statuettes de cire qui représentent la reine et le roi fratricide

12 "C'est son baptême de poète ! et cette comédienne le lui apporte, ainsi enveloppé, de la première scène de Londres. (HSPF, p. 55) 
28

(HSPF, p. 25) illustrent les rapports entre Hamlet et le nouveau couple royal.

Le drame familial de l'hypotexte est donc conservé et présenté dès les premières pages de la nouvelle.

De plus, par une substitution discrète, Laforgue lie Ophélie à Hamlet, par Yorrick interposé. En effet, dans la scène du cimetière, regardant le crâne de Yorrick, Hamlet réfléchit sur la vie et se remémore les paroles du fou de cour:

II y avait une langue là-dedans; ça grasseyait: "Good night, Ladies, good night, sweet ladies! Good night, good night! " (HSPF, p. 46, souligné dans le texte)

Il prête ainsi à Yorrick une réplique qui, dans l'hypotexte, revient à Ophélie ${ }^{13}$. Or, si Yorrick et Ophélie sont ainsi frère et sœur par la folie, et si Hamlet est le frère de Yorrick, Ophélie et Hamlet sont probablement parents...

Enfin, la nouvelle de Laforgue contient aussi une expansion déroutante où Hamlet saccage la nature, arrache les yeux de ses victimes, tord le cou d'un canari, etc.

Folie destructrice dirigée contre la nature pour des raisons philosophiques (l'allégresse de constater que la Justice n'est qu'un mot peut-être, $H S P F$, p. 38) ou encore dirigée contre le père nourricier. Hamlet, chez Laforgue, a en effet un père nourricier qui est «garde» dans «un rendez-vous de chasse" (HSPF, p. 61).

À y regarder de plus près, on constate donc que la nouvelle de Laforgue raconte l'histoire d'un Hamlet qui n'en finit plus de se trouver des parents. Feu le roi et la reine d'abord; un frère, une vraie mère (et une "sœur" de pensée: Ophélie), ensuite; un père nourricier, enfin.

En dépit de tous ces parents, Hamlet se cherche un «Maître»:

Et si je nommais, moi, mon sacré Maître, mon universel

Maître! (HSPF, p. 36)

Ici, l'ambiguïté du «moi " en apposition peut aussi laisser croire que Hamlet se nomme lui-même « Maître». Possibilité

13 Réplique exacte dans la version anglaise: William Shakespeare, The Tragedy of Hamlet, prince of Denmark, Act III, scene 1. 
cohérente, puisque, plus loin dans le texte, il se cherche un nouveau nom, un "nom de guerre» (HSPF, p. 57).

Pris dans son rôle de personnage, Hamlet n'a toutefois pas droit à l'anonymat, ni à l'autonomie. Avec Laforgue, il aura au moins frôlé au plus près cette liberté.

En démotivant son personnage et en (se) jouant de l'hypotexte, Laforgue opère une dévalorisation ironisante du Hamlet de Shakespeare. Dévalorisation sans méchanceté, mais qui démystifie tout en l'"aggravant " le mystère de Hamlet. Pourquoi donc Hamlet retourne-t-il à la tombe d'Ophélie?

En mettant en scène la relation hypertextuelle, Laforgue démultiplie en un miroir supplémentaire cette histoire déjà marquée par la mise en abyme. Il s'agit d'un nouveau regard. L'art, la vie, la mort et l'hérédité se projettent alors à tous les niveaux de la nouvelle (la forme, les réflexions du narrateur, l'histoire, etc.)

Laforgue regarde son cube et le sonde longuement, comme il le ferait d'une boule de cristal. Puis, il rompt le charme et remet le cube à sa place, sur l'étagère. Il salue et sort, marchant de son allure traînarde et correcte, correcte et traînarde...

Tout retourné, le cube Hamlet lui jette un regard en coin et murmure à la ronde : "Ce n'est que partie remise."

\section{Indices bibliographiques}

BAJOMÉE, Danielle, «Hamlet, une moralité oubliée de Jules Laforgue", Revue des langues vivantes, $\mathrm{n}^{\circ} 4$, Bruxelles, 1967, p. 386-405.

GENETTE, Gérard, Palimpsestes. La littérature au second degré, Paris, Seuil, coll. «Poétique», 1982, $467 \mathrm{p}$.

LAFORGUE, Jules, «Hamlet ou les suites de la piété filiale*, Moralités légendaires, Paris, Gallimard, coll. * Folio \#, 1977, 243 p.

SHAKESPEARE, William, La tragédie d'Hamlet, prince de Danemark, dans Hamlet, Le roi Lear, Paris, Gallimard, coll. * Folio *, 1978, p. 25-217.

SHAKESPEARE, William, The tragedy of Hamlet, Prince of Denmark, Edinburgh, Turnbull and Spears, 1902, 215 p.

STEINMETZ, Jean-Luc, «Portrait d'Hamlet en Laforgue adulte ou l'art, le rat et le rate ${ }^{\prime}$, Silex, $n^{\circ} 3$, Grenoble, 1977, p. 88-96. 